05,11

\title{
Критический индекс восприимчивости 1D-изинговского ферромагнетика, замкнутого в кольцо
}

\author{
() Ж.В. Дзюба, В.Н. Удодов \\ Хакасский государственный университет им. Н.Ф. Катанова, \\ Абакан, Россия \\ E-mail: dudareva82@gmail.com
}

(Поступила в Редакцию 30 августа 2017 г.)

\begin{abstract}
Методом Монте-Карло выполнены исследования критического поведения одномерной ферромагнитной модели Изинга с учетом взаимодействия вторых, третьих соседей и четырехчастичного взаимодействия. Полученные результаты критической температуры сравнивались с критической температурой квазиодномерного изинговского магнетика $\left[\left(\mathrm{CH}_{3}\right)_{3} \mathrm{NH}\right] \cdot \mathrm{FeCl}_{3} \cdot 2 \mathrm{H}_{2} \mathrm{O}$, с величиной обменного взаимодействия $J / k_{B}=17.4 \mathrm{~K}$. В рамках теории конечномерного масштабирования рассчитан критический индекс восприимчивости. Показано, что значения индекса восприимчивости для одномерной модели Изинга с периодическими граничными условиями значительно меньше известных значений индексов для трехмерных систем. Критический индекс восприимчивости сильно зависит от энергетических параметров, уменьшается с их ростом.
\end{abstract}

DOI: 10.21883/FTT.2018.07.46115.238

В последние десятилетия синтезирован ряд магнитных соединений с сильной одноосной анизотропией, состоящих из одномерных ферромагнитных цепочек [1]. В критической области исследование магнитных свойств одномерных (квазиодномерных) систем экспериментальными методами связано со значительными трудностями [2]. Поэтому информацию о магнитной структуре и кинетических свойствах квазиодномерных магнетиков получают с помощью компьютерного моделирования. В области фазового перехода свойства тела могут быть описаны набором критических индексов, определяющих законы изменения восприимчивости, теплоемкости и других величин, при приближении к точке перехода $[3,4]$. Для одномерной традиционной модели Изинга некоторые термодинамические (статические) индексы известны из точного решения [3].

Статья посвящена исследованию влияния взаимодействия вторых, третьих соседей, четырехчастичного взаимодействия, температуры и размера системы на восприимчивость и критический индекс восприимчивости 1D-изинговского ферромагнетика методом компьютерного моделирования [5].

$\mathrm{B}$ работе [6] исследования магнетика $\mathrm{FeTaC}$, с формулой $\left[\left(\mathrm{CH}_{3}\right)_{3} \mathrm{NH}\right] \cdot \mathrm{FeCl}_{3} \cdot 2 \mathrm{H}_{2} \mathrm{O}$, показали, что он ведет себя как одномерный изинговской ферромагнетик с обменной постоянной $J / k_{B}=17.4 \mathrm{~K}$ [7]. Высокая степень одномерной изоляции, найденная в $\mathrm{FeTaC}$, делает его привлекательным кандидатом для исследования магнетизма в линейной цепи [6]. Модель Изинга позволяет не только описать переход, но и определить температуру фазового перехода. Важной составляющей частью моделей многих физических систем являются граничные условия, мы рассматривали периодические граничные условия (ПГУ, система замкнута в кольцо), которые часто используются в физике твердого тела [8].

Для моделирования магнитного перехода начальное состояние задается антиферромагнитным, обменные силы с течением времени приводят систему в ферро- магнитное состояние. Алгоритм Метрополиса с динамикой „опрокидывания спина“ является вполне разумным приближением к реальной динамике анизотропного магнетика, спины которого связаны с колебаниями решетки [9]. Восприимчивость рассчитывалась после установления в системе равновесия, которое, как считалось, наступает на шаге $\tau+3 \sigma$, где $\tau$ - число шагов Монте-Карло (МК) на спин (в нашем эксперименте $\mathrm{NMCS}=10000)$ за которое система достигнет равновесия (время релаксации), $\sigma$ - среднеквадратичное отклонение времени релаксации в серии параллельных опытов. В данном эксперименте использовалось число параллельных опытов $L=1000$ (на каждом последовательном опыте число реализаций $Q=100)$, что является оптимальным с точки зрения уменьшения погрешности, устранения зависимости времени релаксации от числа реализаций алгоритма и продолжительности работы программы.

Энергия одномерного ферромагнетика типа FeTaC в безразмерных единицах есть [10]

$$
\begin{aligned}
E=\frac{\varepsilon}{|J|}= & -\left(\sum_{i=1}^{N-1} S_{i} S_{i+1}+S_{N} S_{1}\right) \\
& -J_{2}\left(\sum_{i=1}^{N-2} S_{i} S_{i+2}+\sum_{i=1}^{2} S_{N-2+i} S_{i}\right) \\
& -J_{3}\left(\sum_{i=1}^{N-3} S_{i} S_{i+3}+\sum_{i=1}^{3} S_{N-3+i} S_{i}\right) \\
& -J_{1-4}\left(\sum_{i=1}^{N-3} S_{i} S_{i+1} S_{i+2} S_{i+3}+S_{N-2} S_{N-1} S_{N} S_{1}\right. \\
& \left.+S_{N-1} S_{N} S_{1} S_{2}+S_{N} S_{1} S_{2} S_{3}\right)
\end{aligned}
$$

где $E$ и $\varepsilon-$ энергия магнетика в безразмерных и энергетических единицах соответственно, $j_{1}=J-$ 

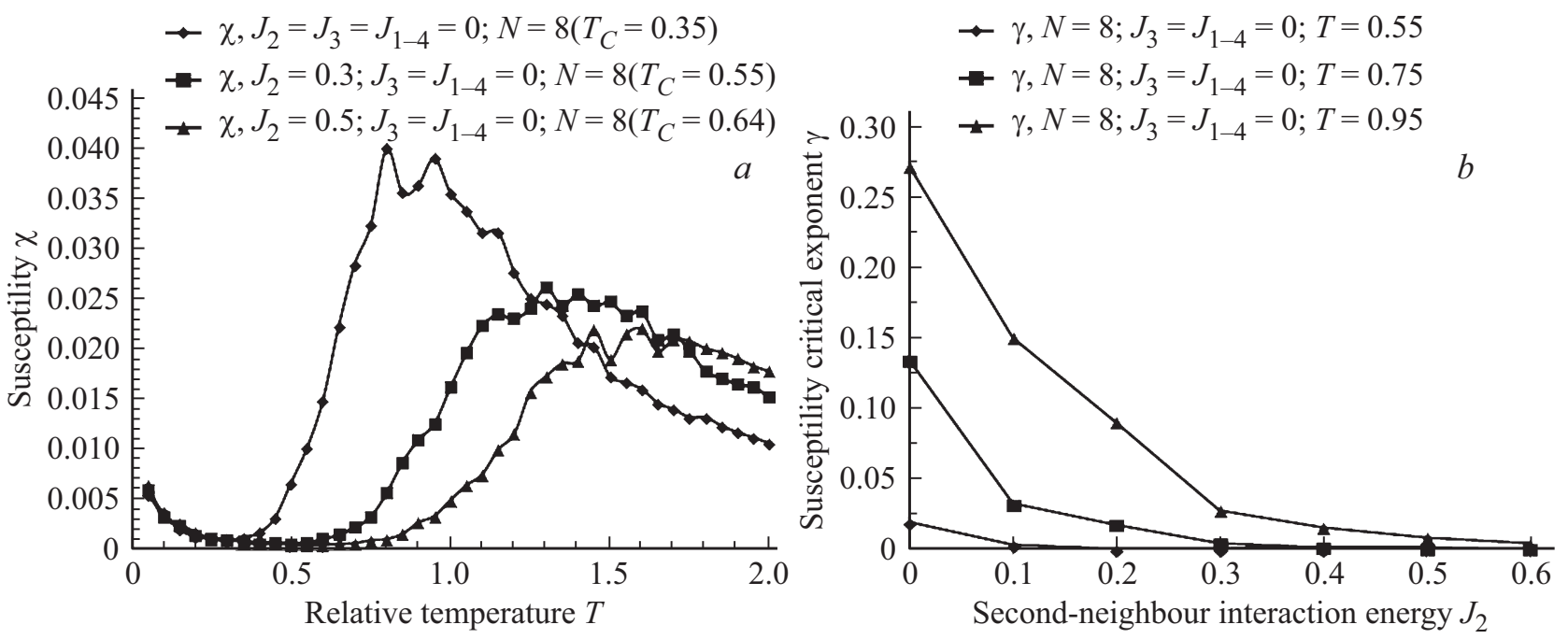

Зависимость $a$ ) восприимчивости $\chi$ от относительной температуры $T$ при разных энергиях взаимодействия $J_{2}$; $\left.b\right)$ Зависимость критического индекса восприимчивости $\gamma$ от энергии взаимодействия вторых соседей $J_{2}$. Относительная погрешность $7 \%$.

энергия взаимодействия между ближайшими соседями (константа обменного взаимодействия $J>0$, известная из эксперимента; энергия минимальна для спинов, направленных в одну сторону), $J_{2}$ - безразмерные энергии взаимодействия вторых соседей, $N$ - количество атомов (узлов), $i$ - номер узла, а $S_{i}-$ спиновая переменная, принимающая значение +1 или -1 .

Метод вычисления восприимчивости основан на связи ее со статистическими флуктуациями намагниченности $M$ в каноническом ансамбле [9], формула для расчета критического индекса восприимчивости методом конечномерного масштабирования имеет вид

$$
\gamma=\ln \left(\chi_{1} / \chi_{2}\right) / \ln \left(N_{1} / N_{2}\right) \cdot v,
$$

где $v-$ критический индекс корреляционной длины. При этом восприимчивость [9] имеет вид

$$
\chi=1 / k_{B} T\left(\left\langle M^{2}\right\rangle-\langle M\rangle^{2}\right),
$$

где $T$ - относительная температура (единицы измерения $j_{1} / k_{B}$; постоянная Больцмана принята за единицу).

Зависимость восприимчивости от температуры имеет максимум, который смещается в большие температуры с ростом энергетических параметров $J_{2}, J_{3}, J_{1-4}$. В работе [11] температурная зависимость восприимчивости тоже достигает максимума и затем линейно уменьшается с понижением температуры, что указывает (как отмечают авторы) на то, что корреляционная длина становится больше, чем среднее расстояние между двумя внутренними дефектами вдоль цепи, поскольку межцепочечные взаимодействия маловероятны. При учете четырехчастичного взаимодействия в области низких температур образуется дополнительный максимум, системе требуется большее количество шагов МК для прихода в равновесие, время релаксации сильно увеличивается. Так, по сравнению со значением при учете взаимодействия вторых и третьих соседей, для четырехчастичного взаимодействия число шагов Монте-Карло было увеличено в 10 раз. Таким образом, проявляется медленная релаксация системы при учете четырехчастичного взаимодействия. Увеличение энергетических параметров вторых, третьих соседей и четырехчастичного взаимодействия уменьшают восприимчивость, температурный максимум становится более пологим, происходит его смещение в большие температуры, см. рисунок, часть $a$.

Восприимчивость и критический индекс восприимчивости сильно зависят от энергетических параметров, уменьшаясь с их ростом, см. рисунок, часть $b$. Так, при $T=0.75$ с ростом энергии взаимодействия вторых соседей от $J_{2}=0$ до $J_{2}=0.6$ для размера системы $N=8$, значения критического индекса восприимчивости принимают значения в диапазоне от $\gamma=0.13 \pm 0.03$ до $\gamma=0.003 \pm 0.0007$ соответственно.

Таким образом, критический индекс восприимчивости сильно зависит от энергетических параметров, уменьшается с их ростом. Так, при учете ближайшего взаимодействия индекс восприимчивости равен $\gamma=0.31 \pm 0.05$, при учете вторых соседей $\gamma=0.12 \pm 0.03$, третьих соседей $\gamma=0.13 \pm 0.05$, и $\gamma=0.19 \pm 0.05$ при учете четырехчастичного взаимодействия. Одномерный индекс восприимчивости для ПГУ существенно меньше известных значений для трехмерной модели Гейзенберга $\gamma=1.39$ [8], и трехмерной $X Y$-модели $\gamma=1.31$ [12]. Полученные данные позволяют провести сравнение с экспериментальными данными для магнитных квазиодномерных систем. Предположим, что максимум на температурной зависимости в нашей работе и в эксперименте совпадает, тогда выражение при учете только ближайшего взаимодействия $k T / J=k T_{\max } / J=0.8$ приравняем к температуре в единицах $\left[k_{B} T / J\right]$ из данных компьютерного эксперимента 1D-модели Изинга при ПГУ. Зная обменную постоянную $J / k_{B}=17.4 \mathrm{~K}$, найдем температуру перехода в упорядоченное состояние в $13.92 \mathrm{~K}$, что больше полученной температуры в работе [7], где переход в упорядоченное состояние наблюдался вблизи температуры $3.1 \mathrm{~K}$. Полученные оценки 
критической температуры ближе к значениям в работе [11], где температура максимума восприимчивости $T_{\max }=11.6 \mathrm{~K}$ хорошо изолированных $1 \mathrm{D}$-цепочек Изинга [13] с эффективным спином $1 / 2$ и обменным взаимодействием $J / k_{B}=19.5 \mathrm{~K}$. Используя данное значения обменного интеграла, получим критическую температуpy $T_{C}=15.6 \mathrm{~K}$, при учете взаимодействия между вторыми соседями $J_{2}=0.3$ получим $T_{C}=25.35 \mathrm{~K}$, между третьими соседями $J_{3}=0.3$ получим $T_{C}=27.3 \mathrm{~K}$, а при учете четырехчастичного взаимодействия $J_{1-4}=0.3$ получим $T_{C}=29.25 \mathrm{~K}$. Поскольку энергия зависит не только от обменного взаимодействия $(J)$, но и от одноионной анизотропии (и от других параметров модели), значение $T_{C}$, рассчитанное по модели Изинга, является приблизительной оценкой.

\section{Список литературы}

[1] N. Hoshino, Y. Sekine, M. Nihei, H. Oshio. Chem. Commun. 46, 6117, (2010).

[2] R. Coldea, D.A. Tennant, E.M. Wheeler, E. Wawrzynska, D. Prabhakaran, M. Telling, K. Habicht, P. Smeibidl, K. Kiefer. Science 327, 177 (2010).

[3] Р. Бэкстер. Точно решаемые модели в статистической механике. Мир, М. (1985). 484 с.

[4] В.Н. Удодов. ФТТ 57, 2018 (2015).

[5] Ж.В. Дзюба, Д.В. Спирин. Программа для ЭВМ „Расчет критического индекса восприимчивости изинговского 1D-магнетика с ПГУ“. Св-во о гос. регистрации программы для ЭВМ № 2017612551 от 29 марта 2017 г.

[6] R.E. Greeney, C.P. Landee, J.H. Zhang, W.M. Reiff. Phys. Rev. B 39, 12200 (1989).

[7] C.P. Landee, R. Kuentzler, J.J.M. Williams. J. Appl. Phys. 67, 5604 (1990).

[8] И.К. Камилов, А.К. Муртазаев, Х.К. Алиев. УФН 169, 773 (1999).

[9] Х. Гулд, Я. Тобочник. Компьютерное моделирование в физике. Ч. 1. Мир, М. (1990).

[10] Ж.В. Дзюба, В.Н. Удодов, Д.В. Спирин. Фундамент. проблемы современ. материаловедения 13, 430 (2016).

[11] R.-M. Wei, F. Cao, J. Li, L. Yang, Y. Han, X.-L. Zhang, Z. Zhang, X.-Y. Wang, Y. Song. Sci. Rep. 6, 24372 (2016).

[12] M. Campostrini, M. Hasenbusch, A. Pelissetto, E. Vicari. Phys. Rev. B 74, 144506 (2006).

[13] T.F. Liu, D. Fu, S. Gao, Y.Z. Zhang, H.L. Sun, G. Su, Y.J. Liu. J. Am. Chem. Soc. 125, 13976 (2003).

Редактор Ю.Э. Китаев 